\title{
Hemodynamics and nociception resulting from Oro-Tracheal Intubation : an observational prospective study
}

\author{
Bouzit Z." , Grati A.H., Cherait C., Abdi W., Haouache H., Dhonneur G.F. \\ Anesthesia and Intensive Care Medicine Department
}

Henri Mondor University Hospital of Paris, Paris 12 School of Medicine, Créteil, France

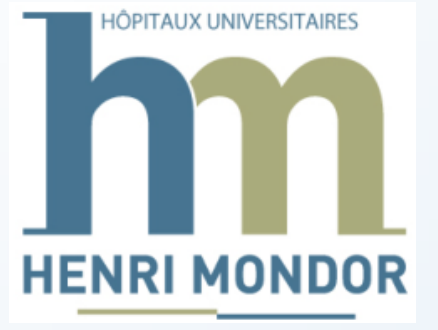

Introduction: Oro-Tracheal Intubation (OTI) is thought to be one of the highest nociceptive stimulation but this assertion has never been clearly proved. We tightly monitored hemodynamics (HD), nociception, and depth of anesthesia during OTI with the aim of pragmatically revisiting this dogma.

Methods: Consenting informed patients scheduled for elective cardiac or vascular surgery were included in this observational prospective study. After admission into the operating room, invasive Blood Pressure (BP), Bis-spectral (BIS) and Analgesia Nociception Index (ANI) monitoring systems were installed and set-up. The induction procedure of anesthesia with target-controlled infusion was standardized using sufentanil and propofol. Timed OTI maneuver $(<30$ s) truncated in 3 periods $(P)$ of predefined duration was performed after monitored deep neuromuscular blockade (atracurium) was installed. P1 (10-15s) corresponded with direct laryngoscopy, P2 (5-10s) with Tracheal Tube (TT) manipulation, and P3 (<5s) with TT cuff inflation. Recorded HD, BIS and ANI data were analyzed at 6 predefined time points. T0: baseline values after monitors installation, T1: post induction of anesthesia and just before OTI, T2: end of P1, T3: end of P2, T4: end of P3, T5 and T6, respectively 1 and 5 min after the end of OTI. Recorded parameters evolution was compared using appropriate statistics.

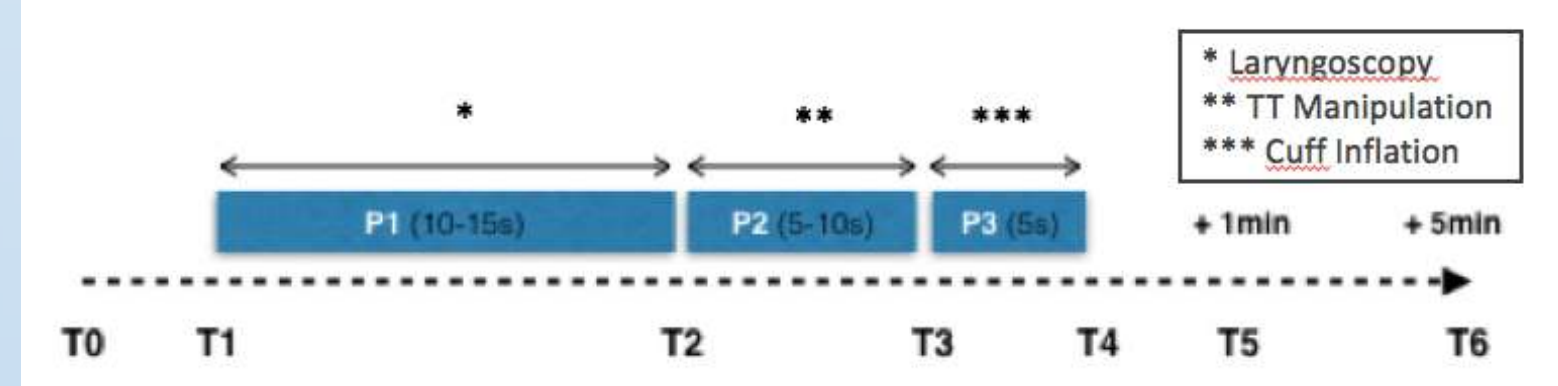

TO = baseline,

$T 1=$ post induction of anesthesia, before OTI,

$T 2=$ end of $P 1, T 3=$ end of $P 2, T 4=$ end of $P 3$,

T5 / T6 = 1 minute $/ 5$ min after the end of OTI.

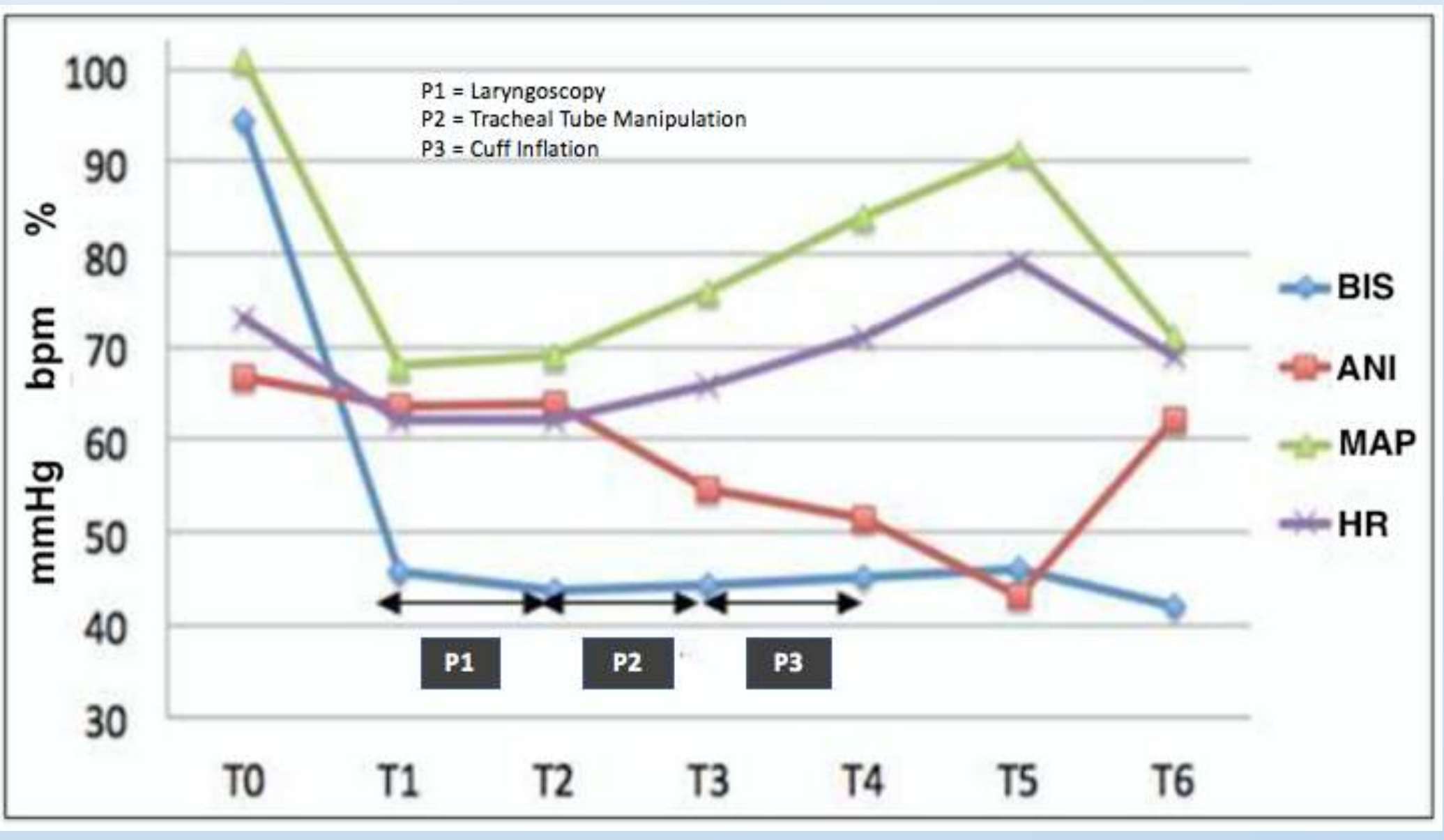

Monitored parameters evolution (mean) during OTI

Results: Thirty nine patients were included, but 4 were secondarily excluded because of OTI lasting more than 30 s. $\mathrm{P} 1$ is characterized by a remarkable stability of all measured parameters. P2 and even more P3 are associated with intense and significant HD and ANI variations. ANI variations are completely superimposed, but opposite, to those of MAP and HR. BIS variations during TI were negligible.

Discussion: Our data suggest that laryngoscopy may not be the most intense nociceptive stimulus applied to patients during OTI. Both laryngeal and/or tracheal stimulation resulting from tracheal tube passage and cuff inflation seem to promote very intense nociception. Interinstingly, such phenomenon could be erased by either laryngeal mucous topicalization or superior laryngeal nerve bilateral blocks 